\title{
Miniaturised Optoelectronic Tweezers Controlled by GaN Micro Light Emitting Diode Arrays
}

\author{
Alicja Zarowna-Dabrowska ${ }^{1,2}$, Steven L. Neale ${ }^{2}$, David Massoubre ${ }^{1}$, Jonathan McKendry ${ }^{1}$, Bruce R. Rae ${ }^{3}$, \\ Huabing Yin ${ }^{2}$, Robert K. Henderson ${ }^{3}$, Mervyn J. Rose ${ }^{4}$, Martin D. Dawson ${ }^{1}$, Jonathan M. Cooper ${ }^{2}$ and Erdan Gu

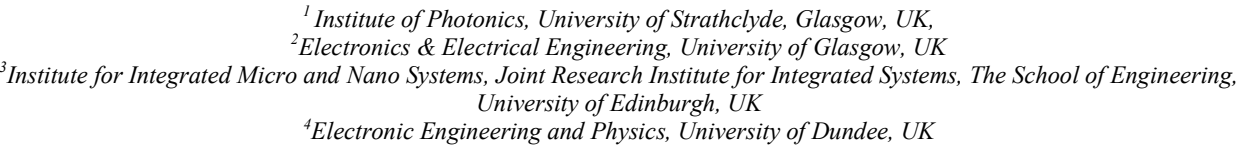

Institute of Photonics, University of Strathclyde, Glasgow, UK,

${ }^{2}$ Electronics \& Electrical Engineering, University of Glasgow, UK

${ }^{3}$ Institute for Integrated Micro and Nano Systems, Joint Research Institute for Integrated Systems, The School of Engineering, University of Edinburgh, UK

${ }^{4}$ Electronic Engineering and Physics, University of Dundee, UK

\begin{abstract}
CMOS-controlled GaN micropixel light emitting diode arrays have been used to create light patterned electrodes in a miniaturized optoelectronic tweezers device. We present this micro-system's capabilities for manipulating micro-particles including beads and cells.
\end{abstract}

\section{INTRODUCTION}

The ability to move and control the position of micro-particles is essential for numerous cell biology experiments. There are many competing micromanipulation techniques being actively researched, including mechanical manipulation using AFM tips, optical tweezers, dielectrophoresis, magnetic traps and acoustic traps. Each technique has distinguishing advantages and disadvantages with some offering higher forces whilst others offer more precise manipulation. Recently developed technique, Optoelectronic Tweezers (OET), uses a light patterned photoconductor to provide real time control over the positioning of electric fields. OET has advantages over traditional dielectrophoresis as the field can be repositioned and more particles can be manipulated individually and in parallel than with AFM, magnetic tweezers or optical tweezers [1]. The OET traps have been compared to optical traps and have found to be 470 times stiffer for a similar light intensity [2]. Disadvantages of OET are that it is a $2 \mathrm{D}$ force and the trapping is not as strong as with an AFM tip or with magnetic traps. There are also limitations on the liquids that the particles can be suspended in. The light pattern that creates the virtual electrode in an OET device has been created to date by either using a Digital Micro-mirror Device (DMD) [1] or using an LCD display [3]. However, these DMD and LCD devices are quite bulky. In this work, by using integrated CMOS controlled micro-Light Emitting Diode arrays (micro-LEDs) to create micro-light patterns, a miniaturised OET device is demonstrated.

\section{MINIATURISED OET DESIGN AND STRUCTURE}

CMOS-controlled micro-LEDs have 'micro-disk' pixel sizes of ten to a few tens of microns producing a light pattern with precise spatial control [4]. This allows one to generate a light pattern directly with the light source rather than using a spatial light modulator. This facilitates integration, offers independent pixel control and the possibility of switching modes of operation of the device (e.g. CW to sub-nanosecond pulsing for combined manipulation and time-resolved fluorescence analysis). Figure 1a shows such a miniaturised OET device. The OET device relies on a layer of amorphous silicon (a-Si) at the bottom of the sample chamber. In order to get the best image of the particles, we choose to view the sample chamber from the top and place the micro-LEDs underneath to illuminate the a-Si layer. The light from the micro-LEDs is focused onto the a-Si layer by a low-cost lens, $6 \mathrm{~mm}$ diameter, NA 0.55 (Geltech $^{\mathrm{TM}}$, Thorlabs), fixed on the top of microLEDs, as shown in figure $1 \mathrm{~b}$.
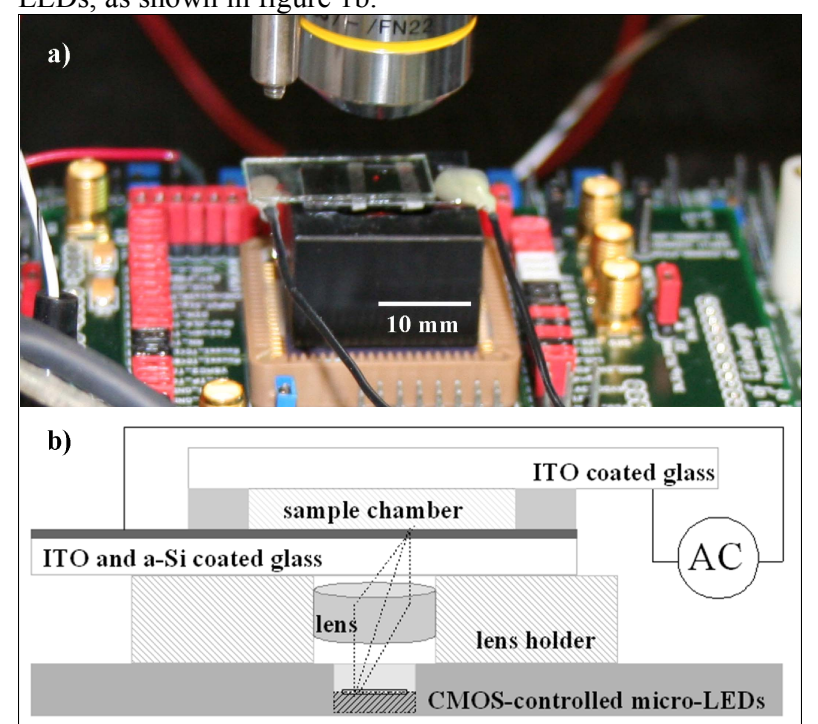

Figure 1. a) Picture of the integrated miniaturised OET device; b) Schematic of the vertical section of the device.

The micro-LED chips, each consisting of $8 \times 8$ arrays of micropixels flip-chip bump bonded to a CMOS control backplane, were fabricated with different emission wavelengths i.e. $450 \mathrm{~nm}$ and 520nm, with different pixel sizes [4]. The typical turn-on voltage, drive current and power output of a LED pixel depend on semiconductor wafer characteristics and pixel size [5]. We have used blue $(450 \mathrm{~nm})$ and green $(520 \mathrm{~nm})$ devices with pixel sizes from $14 \mu \mathrm{m}$ to $84 \mu \mathrm{m}$ (by step of $10 \mu \mathrm{m}$ ) and a center-tocenter pitch of $200 \mu \mathrm{m} .450 \mathrm{~nm}$ devices have a typical turn-on voltage of $3.0 \mathrm{~V}$ and can produce an output power up to $4.5 \mathrm{~mW}$ $(84 \mu \mathrm{m}$ pixel at a drive current of $140 \mathrm{~mA})$ [6]. 520nm microLEDs have a typical turn-on voltage of $4.2 \mathrm{~V}$ and an average 
output power of $300 \mu \mathrm{W}$ at $21 \mathrm{~mA}$ for a $74 \mu \mathrm{m}$ pixel size. In the literature, we have found that wavelengths between $520 \mathrm{~nm}$ and $560 \mathrm{~nm}$ give the best collection efficiency for solar cells made from a-Si [7], suggesting that green micro-LEDs may be most effective for exciting a-Si photoconductive electrodes. Indeed, we found that blue devices produced no detectable particle movement, but that green devices were successful, as described below. In these experiments, the a-Si thickness was $300 \mathrm{~nm}$ and was free of observable defects. For trapping, we used $10 \mu \mathrm{m}$ polystyrene beads in low concentration $\mathrm{KCl}$ solution and Chinese Hamster Ovary (CHO) cells in a solution of sugars (0.3\% Dextrose, $8.5 \%$ Sucrose). We fixed CMOS driver at its maximum output voltage of $4.95 \mathrm{~V}$. For a fixed bias, the current and power output of LED pixels decrease when reducing the pixel size due to the increase of pixel's differential resistances [5]. Illuminated by using green LED pixels, $0.3 \mathrm{~W} / \mathrm{cm}^{2}$ power density was measured at the bottom surface of the sample chamber. In addition, to achieve particle trapping, a range of parameters related to photoconductive cell operation, such as AC drive frequency, voltage and solution conductivity, have been optimized.

\section{EXPERIMENTAL RESULTS AND DISCUSSION}

By using 300nm thick a-Si with green LED illumination, it was found that the best conditions for trapping are: frequency of 10 $\mathrm{kHz}$ and solution conductivity of $10 \mathrm{mSm}^{-1}$ for $10 \mu \mathrm{m}$ polystyrene beads and $1 \mathrm{mSm}^{-1}$ for CHO cells. The best results of particles trapping have been achieved with LED pixels from $44 \mu \mathrm{m}$ to $74 \mu \mathrm{m}$ in diameter. Figure 2 shows examples of polystyrene beads and $\mathrm{CHO}$ cell trapping with one LED pixel in a green micro-LED array.

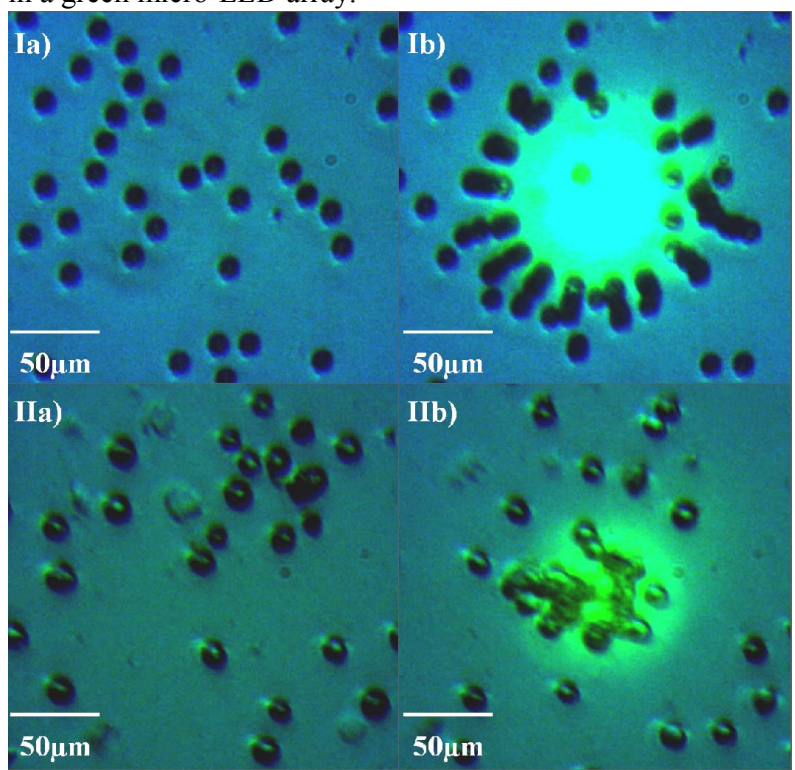

Figure 2. Ia and IIa were taken with the LEDs turned-off; Ib and IIb were captured 60 seconds after a pixel had been turned on; Ib) Trapping beads with $74 \mu \mathrm{m}$ diameter pixel, at $20 \mathrm{~V}$ peak to peak voltage, Ilb) Trapping cells with $54 \mu \mathrm{m}$ pixel at $5 \mathrm{~V}$ peak to peak voltage.
By inspecting the behaviour of particles (beads or cells), we have found that the mechanisms responsible for trapping were mainly light-induced AC electro-osmosis (LACE), which induced liquid flow towards the illuminated area and dielectrophoresis (DEP). Driven by LACE, the particles may move from a long distance. DEP action, however, only dominates within a shorter distance from the illuminated areas. The permittivity of particles relative to the solution in which they were suspended is an important parameter to determining their trapping behaviour. Polystyrene beads have a lower permittivity and $\mathrm{CHO}$ cells have a higher permittivity compared with the respective solution. Consequently, beads 'felt' negative DEP, attracting them to the border of the pixel, where the electrical field is weakest and their potential energy is minimal. Cells, however, 'felt' positive DEP and have been attracted to the centre of the illuminated area, where the electrical field is strongest. The images shown in figure 2 clearly demonstrate these trapping characteristics. In order to further understand these phenomena, we are carrying out the simulation of the electrical field distribution.

Due to the high efficiency of the a-Si at green wavelength, lower light power is required for particle trapping. The power density measured at the bottom surface of the sample chamber was $0.3 \mathrm{~W} / \mathrm{cm}^{2}$. This power density is much less than the 250 $\mathrm{W} / \mathrm{cm}^{2}$ used previously with a $625 \mathrm{~nm}$ commercial diode [1], substantially decreasing the light radiations to the trapped biological samples. In addition, our micro-pixellated light source is powered and controlled by a computer through a USB connection thanks to its low power consumption. The trapping areas created in our system are useful for trapping many cells and can be further reduced by de-magnifying the optical pattern.

\section{CONCLUSIONS}

In summary, we have demonstrated the performance of a miniaturised OET device. The trapping of polystyrene beads and cells has been achieved by switching on and off individual pixels with lower power than used in previous studies. By combining this CMOS-controlled micro-LED array with smaller imaging system, this technology has the potential to create portable and low-cost multi-functional OET device.

\section{REFERENCES}

P. Y. Chiou, A. T. Ohta, and M. C. Wu, Nature, vol. 436, pp. 370372, 2005.

S. L. Neale, M. Mazilu, J. I. B. Wilson, K. Dholakia, and T. F. Krauss, Optics Express, vol. 15, pp. 12619-12626, 2007.

H. Hwang, Y. J. Choi, W. Choi, S. H. Kim, J. Jang, and J. K. Park, Electrophoresis, vol. 29, pp. 1203-1212, 2008.

B. R. Rae, K. R. Muir, Z. Gong, J. McKendry, J. M. Girkin, E. Gu, D. Renshaw, M. D. Dawson, and R. K. Henderson, Sensors, vol. 9 , pp. 9255-9274, 2009.

Z. Gong, S. R. Jin, Y. J. Chen, J. McKendry, D. Massoubre, I. M. Watson, E. Gu, and M. D. Dawson, Journal of Applied Physics, vol. 107, 2010.

J. McKendry, R. P. Green, A. E. Kelly, Z. Gong, B. Guilhabert, D. Massoubre, E. Gu, and M. D. Dawson, IEEE Photonics Technology Letters, 2010, to be published.

D. E. Carlson and C. R. Wronski, Applied Physics Letters, vol. 28, pp. 671-673, 1976. 